\title{
Raindrop Erosion of Composite Materials: Some Views on the Effect of Bending Stress on Erosion Mechanisms
}

\author{
Kieran Pugh ${ }^{1}$ (1) $\cdot$ Ghulam Rasool $^{1} \cdot$ Margaret M. Stack $^{1}$
}

Received: 28 January 2019 / Revised: 25 February 2019 / Accepted: 13 March 2019 / Published online: 2 April 2019

(c) The Author(s) 2019

\begin{abstract}
This paper represents the investigation of rain erosion on wind turbine blade materials under load in the simulation of onshore and offshore environmental conditions. The experimental work was carried out on a whirling arm rig with the material under a static 3 point bend to simulate large multi-megawatt wind turbine blades flexing during operation. This experiment was run with both fresh water and salt water to simulate onshore and offshore turbines. The results showed that the effects of a pre-stress on the samples resulted in a higher degradation rate following rain drop erosion. The microscopic analysis of the samples exposed to pre-stress identified distinctive surface features which has been termed a surface impact circular deformation. These features showed signs of cracking which enhanced the erosion rate. The pre-stressed samples also encountered a larger crossover in erosive mechanisms of abrasion and direct impacts; this was theorised to be due to the material being close to its yield stress and more likely to plastically deform.
\end{abstract}

Keywords Wind energy $\cdot$ Wind turbines $\cdot$ Erosion $\cdot$ Rain erosion $\cdot$ Droplet impact $\cdot$ Wear maps

\section{Introduction}

Rotor diameters of commercial scale, multi-megawatt, wind turbines have grown in size dramatically [1]. This increase in blade length has progressed due to; advances in blade technology, optimising aerodynamic profiles and improvements with the materials. This common trend is to increase power output and efficiency; the result is some turbine blades being in excess of $80 \mathrm{~m}$ long ( $160 \mathrm{~m}$ diameter). In such structures, very significant stresses and strains are being applied to the material due to the static weight of the larger blades loading and high tangential tip velocities. The purpose of this paper is to investigate the effect of the loading to uncover if there is more considerable erosion due to rain impacts when there is a bending stress on the blade due to its size and weight.

Kieran Pugh

Kieran.pugh@strath.ac.uk

Ghulam Rasool

ghulam.rasool@strath.ac.uk

Margaret M. Stack

margaret.stack@strath.ac.uk

1 Department of Mechanical and Aerospace Engineering, University of Strathclyde, James weir Building, 75 Montrose Street, Glasgow G1 1XJ, UK
It is becoming increasingly popular within countries to aim for a more renewable production of electricity. This is because of climate change and the increasing limitations of fossil fuels. The Nordic countries; Norway, Denmark and Sweden are leaders in the industry. For example, Norway is becoming exceedingly close to becoming a country that produces $100 \%$ of its electricity from renewable sources [2]. A large producer of this electricity is from wind power contributing 1.9 TWh [3]. In order to be able to generate this magnitude of energy from wind, major innovations were made with both design and materials. LM power are leading the way in terms of wind power technology and have recently unveiled their newest addition, an $88.4 \mathrm{~m}$ long blade made from a new hybrid material consisting of carbon and glass fibres infused within a matrix [4]. It is generally assume that if the length of a wind turbine blade is doubled, the wind turbine produces four times as much power; however, in this case, the mass of the blades increases by a factor of eight [4]. This can lead to the assumption that wind energy follows Moore's law in that there will be a limit of blade length and technology above which any further improvement would not be beneficial due to the small increase in energy production against the cost of achieving it. To date, blades are still increasing in size. This is producing more engineering challenges [1], one of which is the stresses the blade must 


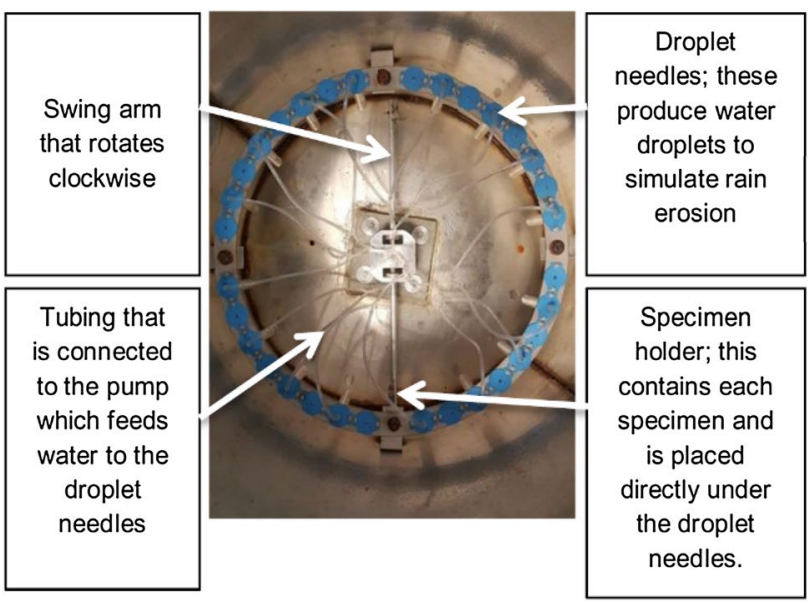

Fig. 1 Rain drop erosion experiment rig at The University of Strathclyde

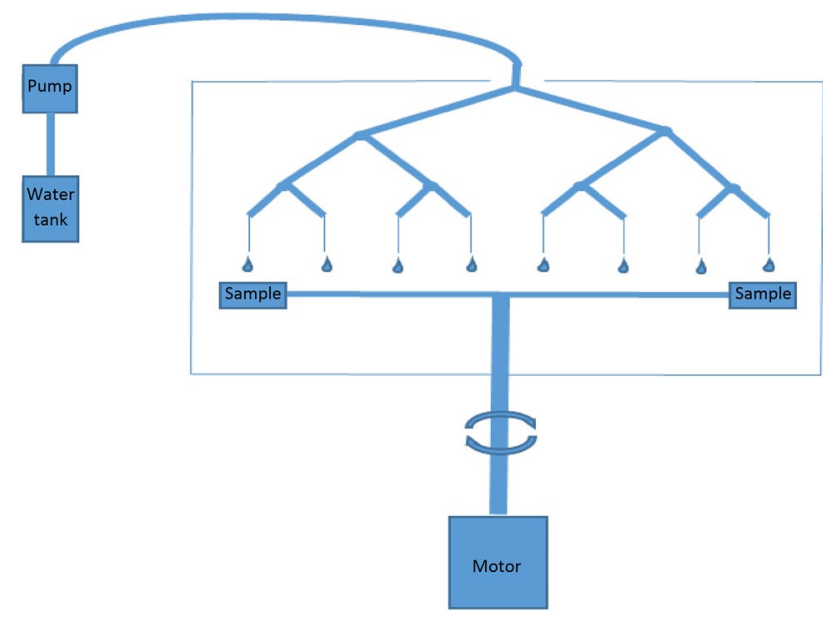

Fig. 2 Line drawing of experimental rig

be able to withstand due to the severe flex the blade endures whilst in operation [5-7].

Another significant engineering issue is the material degradation during the lifetime of the wind turbine in operation. This can be caused by lots of different factors including; UV damage, hailstone erosion, sea water drift and rain erosion. In this investigation, the focus will be on sea water drift and rain erosion as this is what has been tested within the previous experimental portion.

For wind turbines to work most efficiently they must be situated in predominantly windy environments, however, these conditions pose additional challenges such as large amounts of rainfall that comes with these areas, changing temperatures and their remoteness causing difficulties in access $[8,9]$. In Scotland, the largest windfarm in Europe is present i.e. the Whitelee windfarm. This windfarm consists of 215 Siemens and Alstom wind turbines with a total capacity of $539 \mathrm{MW}$. The location of this wind farm is just 10 miles south of Glasgow and the conditions are ideal for wind energy generation as it is located $300 \mathrm{~m}$ above sea level [10]. Nevertheless, conditions like these come at a disadvantage as the environment is very harsh and can be extremely erosive to the blades. With larger turbine blades, the tips are travelling much faster and the impact from a rain droplet will be significant resulting in increased erosion on the leading edge [11].

This erosion can be a disruption in many ways, including more frequent downtime of the blades for visual inspection. This will mean repairing the blade, if necessary, to maintain structural integrity and also to ensure efficiency of the power generation. Leading edge erosion results in an increase in the blade drag. From the literature it shows with only "mild erosion" there is a 6\% increase in drag and for "severe erosion" there could be an increase of $500 \%$ drag; this can result in a loss of $25 \%$ of power generation annually [12].

Such effects can be even more extreme in the offshore wind turbines with presence of sea spray and a corrosive atmosphere. Repair and maintenance also present challenges due to access being limited. Despite this, off shore wind farms are becoming more prevalent due to planning issues associated with land based turbines. For these reasons, there is an increasing interest in offshore wind turbine blade erosion.

In this paper, two potential issues of increased erosion due to stress and sea water are being brought together in order to try and understand their combined effects effect and whether they have a synergetic relationship. This research is of importance now as it encompasses both early wind turbines, primarily land based, and the new offshore structures. It considers the current operating wind turbines and whether approximate quoted life expectancy from the manufacture is accurate and investigates whether stress on the blade has a considerable effect on the leading edge erosion. It also addresses future blade development as these two factors of flexural stress and leading-edge rain erosion will be more of significance than the older smaller blades. This investigation will also consider the effect of both factors in off shore and onshore conditions in order to research the effects in both locations.

\section{Experimental}

\subsection{Methodology and Test Apparatus}

The type of rig used for this experiment was a whirl arm rig shown in Figs. 1 and 2. It involves a motor spinning a shaft which is connected to a central hub to which two samples are connected at a radius from the centre. At the circumference of the swing arm includes needles which provide 
Fig. 3 Percentage mass loss of fresh water erosion with no applied bending stress
Fig. 4 Percentage mass loss of fresh water erosion with an applied bending stress
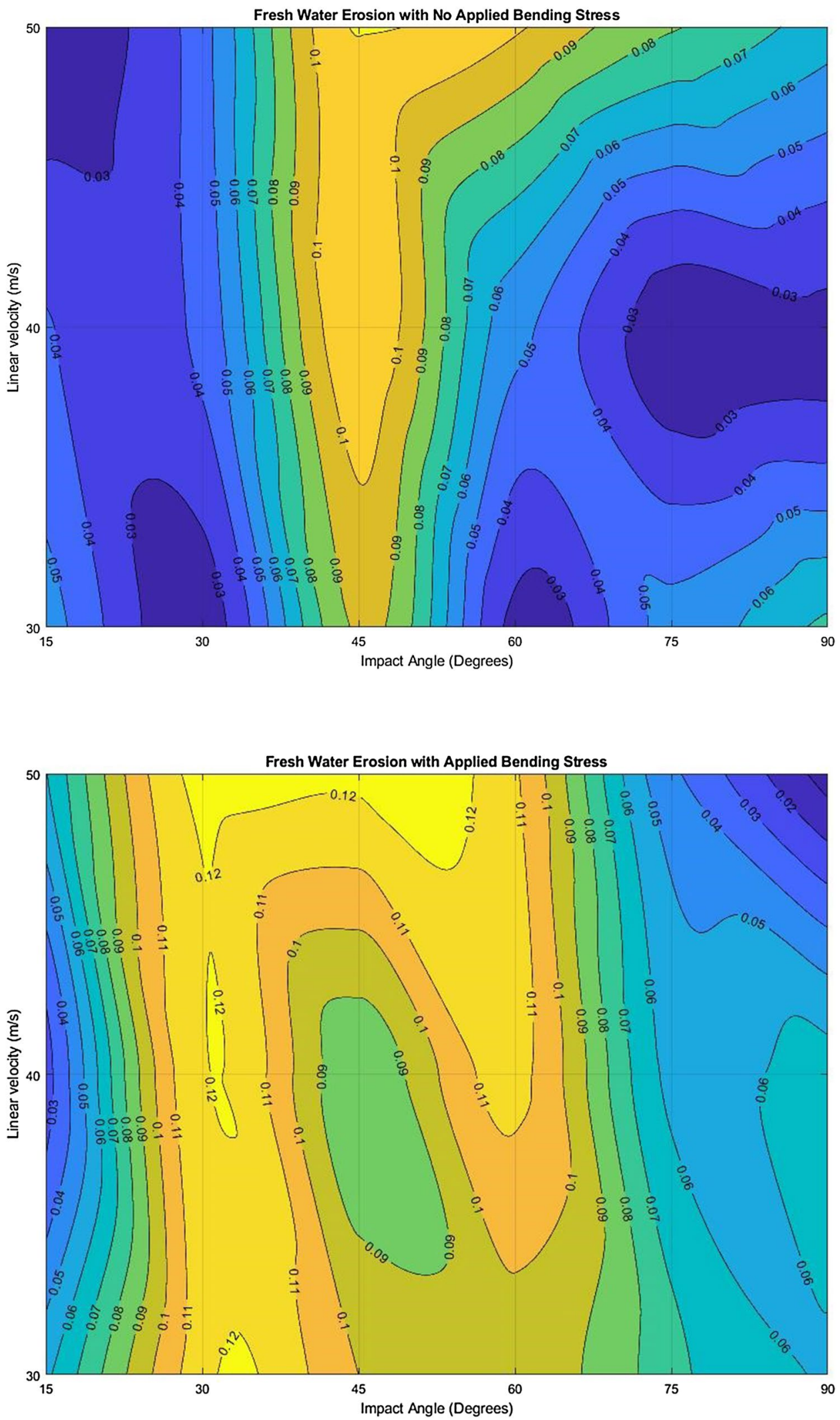
Fig. 5 Percentage mass loss of salt water erosion with no applied bending stress

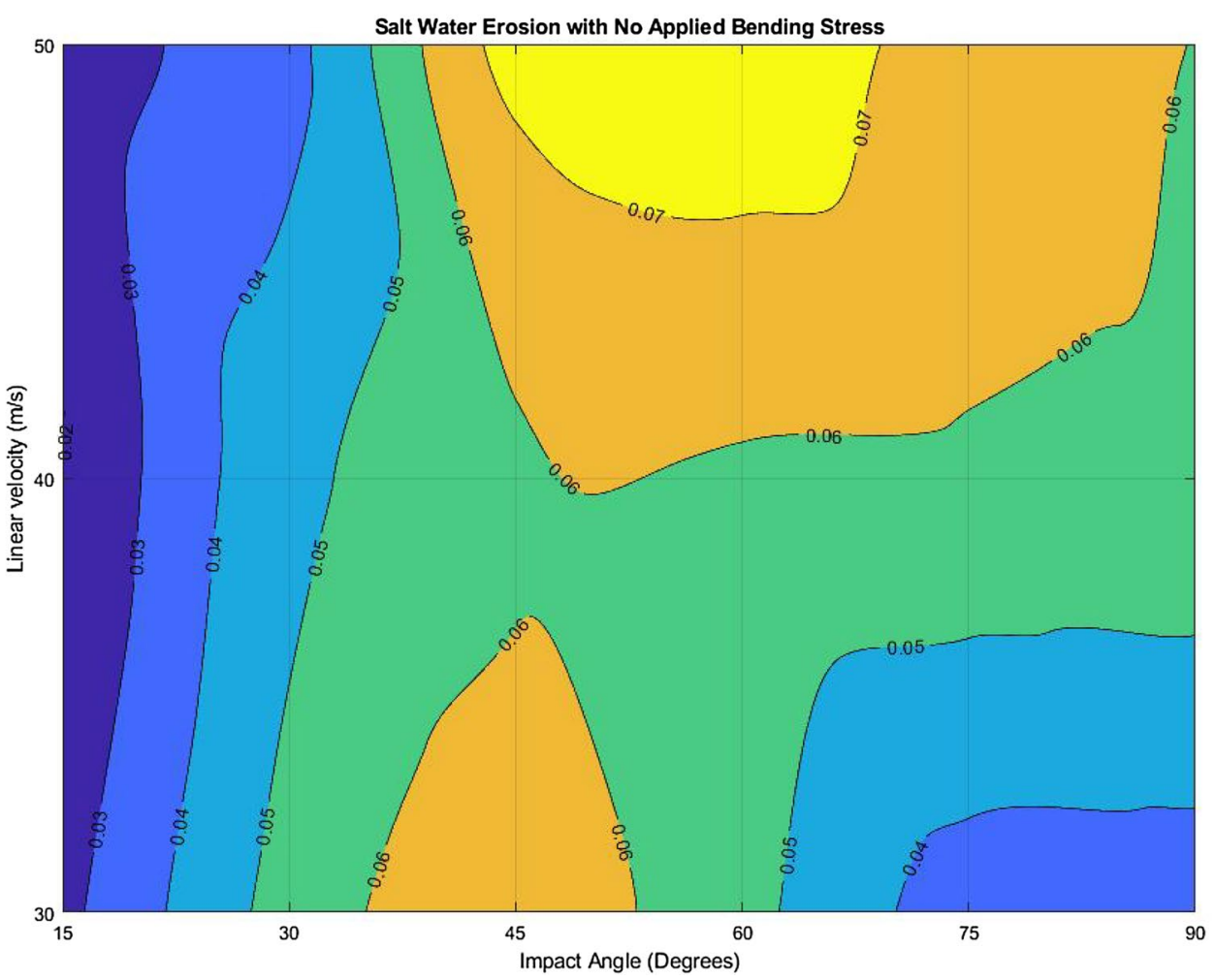

Fig. 6 Percentage mass loss of salt water erosion with an applied bending stress

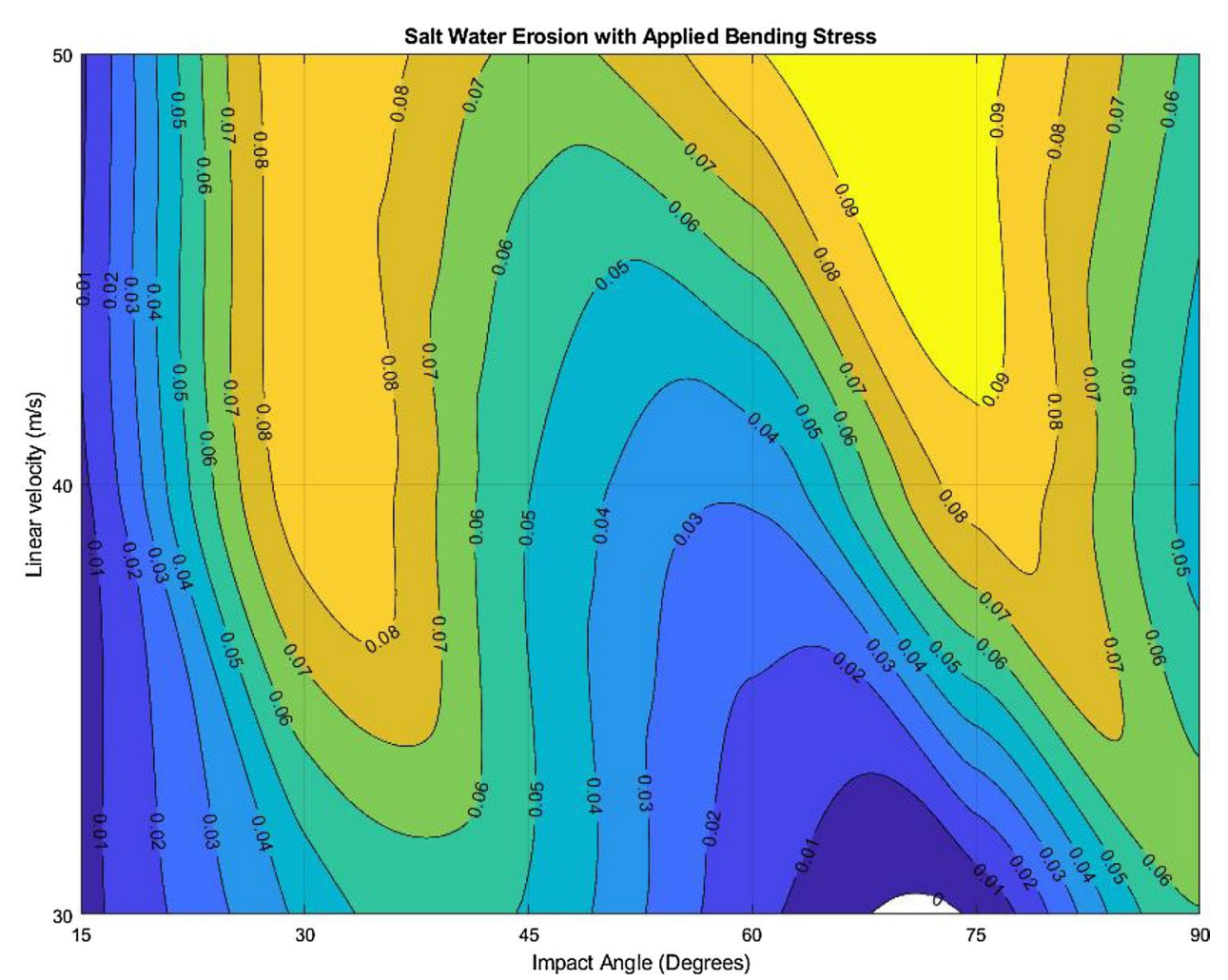




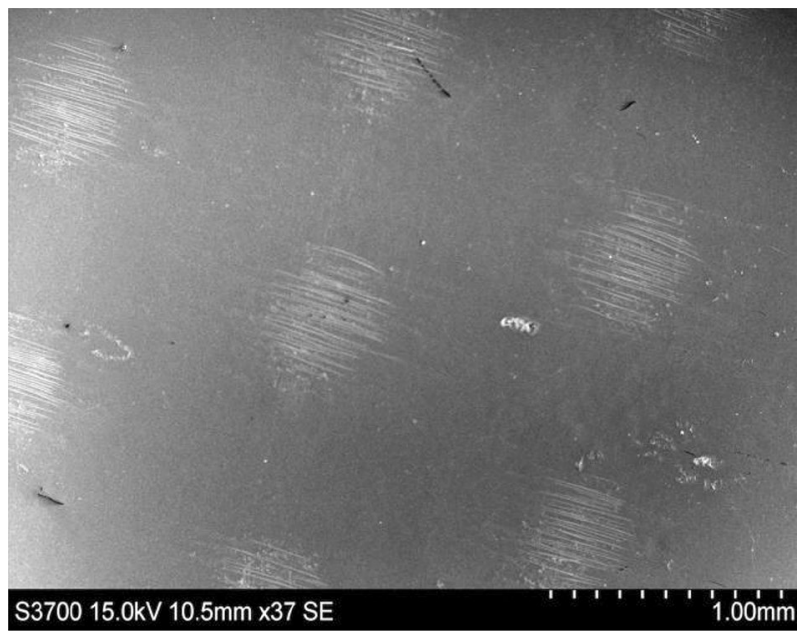

Fig. 7 SEM image of fresh water erosion on an unstressed sample at $30 \mathrm{~ms}^{-1}, 45^{\circ}$

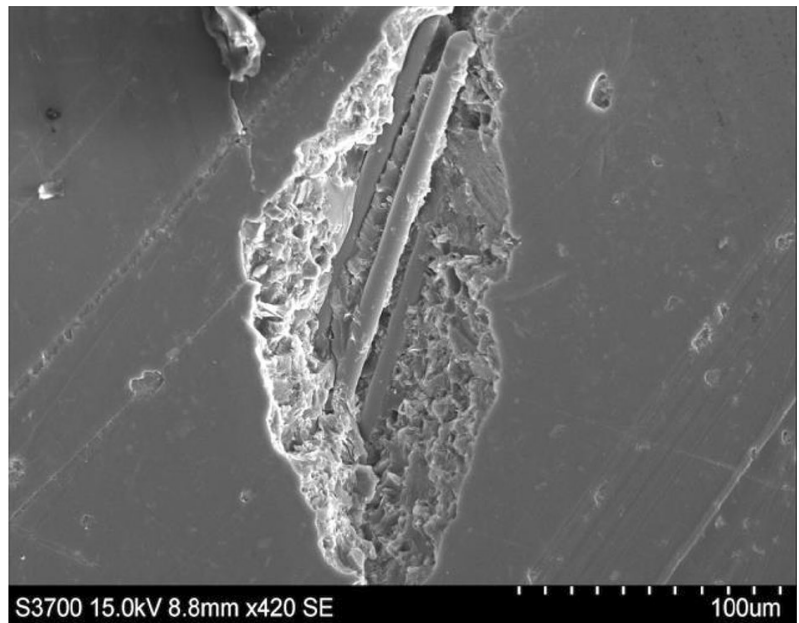

Fig. 8 SEM image of fresh water erosion on an unstressed sample at $40 \mathrm{~ms}^{-1}$ at $30^{\circ}$

water droplets to the rig fed by a pump on the outside of the container.

The experimental rig used for this investigation was an existing setup within the tribology department at The University of Strathclyde. This rig was previously used to collect experimental data for the paper titled "Some Thoughts on Mapping Tribological Issues of Wind Turbine Blades Due to Effects of Onshore and Offshore Raindrop Erosion" [13]. Adjustments were made to this rig to allow a bending stress to be applied to the sample. This was achieved by redesigning the sample holder to pre-stress the sample in a static 3-point bend. The sample holder was then attached to the whirling arm rig to undergo rain erosion while the sample was under stress in the 3-point bend. The flexural stress was calculated by measuring the deflection the sample
Table 1 Running times and distances of experimental rig

\begin{tabular}{ll}
\hline Velocity $\left(\mathrm{ms}^{-1}\right)$ & Experimental time \\
\hline 30 & $108,000 / 30=3600 \mathrm{~s}=60 \mathrm{~min}$ \\
40 & $108,000 / 40=2700 \mathrm{~s}=45 \mathrm{~min}$ \\
50 & $108,000 / 50=2160 \mathrm{~s}=36 \mathrm{~min}$ \\
\hline
\end{tabular}

Table 2 Material properties of G10 Epoxy glass

\begin{tabular}{ll}
\hline Flexural strength $(\mathrm{MPa})$ & 482 \\
Tensile strength $(\mathrm{MPa})$ & 320 \\
Shear strength $(\mathrm{MPa})$ & 131 \\
Chary impact strength $\left(\mathrm{kJ} \mathrm{m}^{-2}\right)$ & 65 \\
Density $\left(\mathrm{g} \mathrm{cm}^{-3}\right)$ & 2 \\
Specific gravity & 1.82 \\
Water absorption $\left(\mathrm{mg} \mathrm{m}^{-2}\right)$ & 8 \\
Hardness, Rockwell $\left(\mathrm{m}^{-\mathrm{scale})}\right.$ & 110 \\
Body colour & Green \\
Standard finish & Stain/glossy \\
\hline
\end{tabular}

was experiencing. Each sample was subjected to approximately $2 \mathrm{~mm}$ of deflection and the flexural stress was then determined depending on the sample's width, length and thickness. Due to the scale of this rig with a radius of only $0.3 \mathrm{~m}$ the samples were required to be small, this resulted in minor changes in the sample size to have a greater effect on the calculated flexural stress.

Before each test was carried out the samples were cut to size at least 2 days prior. On the day of the experiment the samples were weighed using a 5-point scale. The parameter being tested on specific experiments were set accordingly and the water supply to the tank was engaged to allow time for the environment to reach equilibrium. The samples were then inserted, and the experiment began.

After the completion of each experiment the samples were left to dry. The samples were reweighed at least 3 days after testing to ensure they had reached a stable water absorbance and their weights would be comparable.

\subsection{Test Conditions}

The variables tested within this investigation are as follows; Impact velocity $\left(\mathrm{ms}^{-1}\right) 30,40,50$; impact angle (degrees) $15,30,45,60,75,90$; liquid medium as $3.5 \%$ salt solution to mimic salt water and fresh water from a water source to mimic rain. The impact velocity has been changed slightly during this investigation as the sample holders were slightly heavier and thus made the rig more unstable at $60 \mathrm{~ms}^{-1}$ deeming it unable to operate above $50 \mathrm{~ms}^{-1} .20 \mathrm{~ms}^{-1}$ was not investigated as it did not show enough considerable erosion 
Fig. 9 Line graph of fresh water erosion with no bending stress

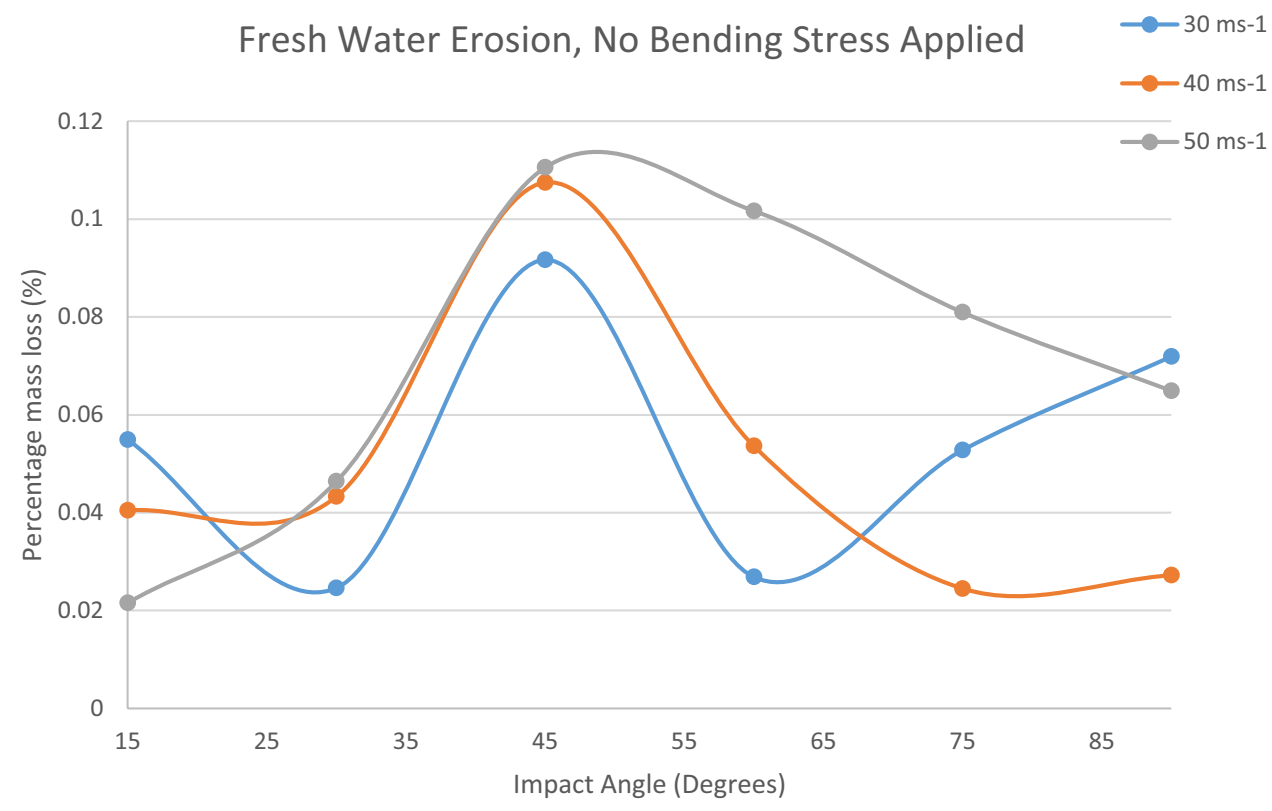

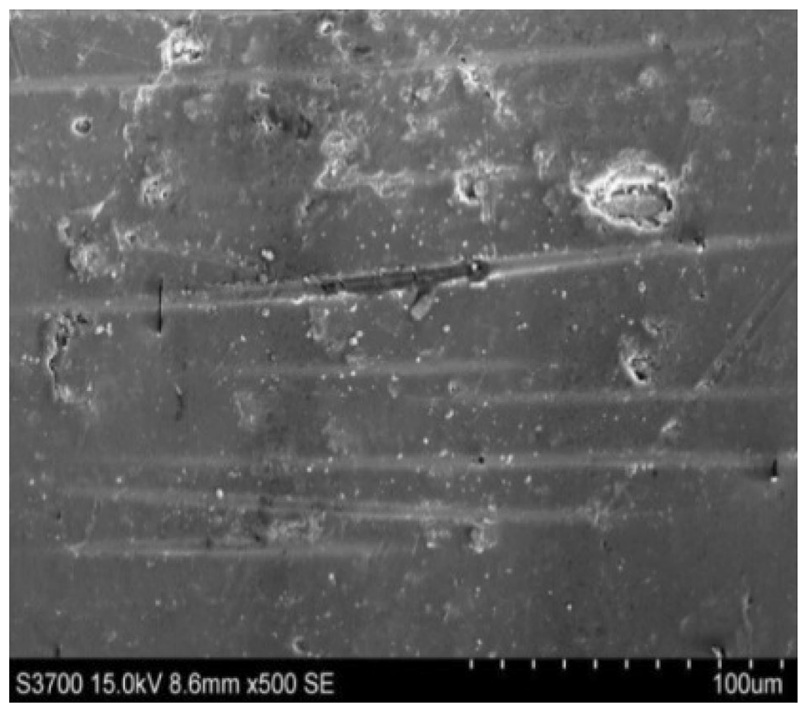

Fig. 11 SEM image of salt water erosion on an unstressed sample at $50 \mathrm{~ms}^{-1}, 45^{\circ}$

the pump at the same setting; the material for the test samples was kept constant using G10 epoxy glass laminate.

The flow rate of the peristaltic pump was calculated to ensure it was consistent with the manufacture claim and the rainfall flow rate. The flow rate was calculated during the test experiments and the weight of the water in the supply water tank for the pump was weighed before and after testing and the time the pump was running for to calculate the flow rate:

$$
V_{\mathrm{i}}=4300.4 \mathrm{~g}=4300.4 \mathrm{ml}
$$


Fig. 12 Line graph of salt water erosion with no bending stress
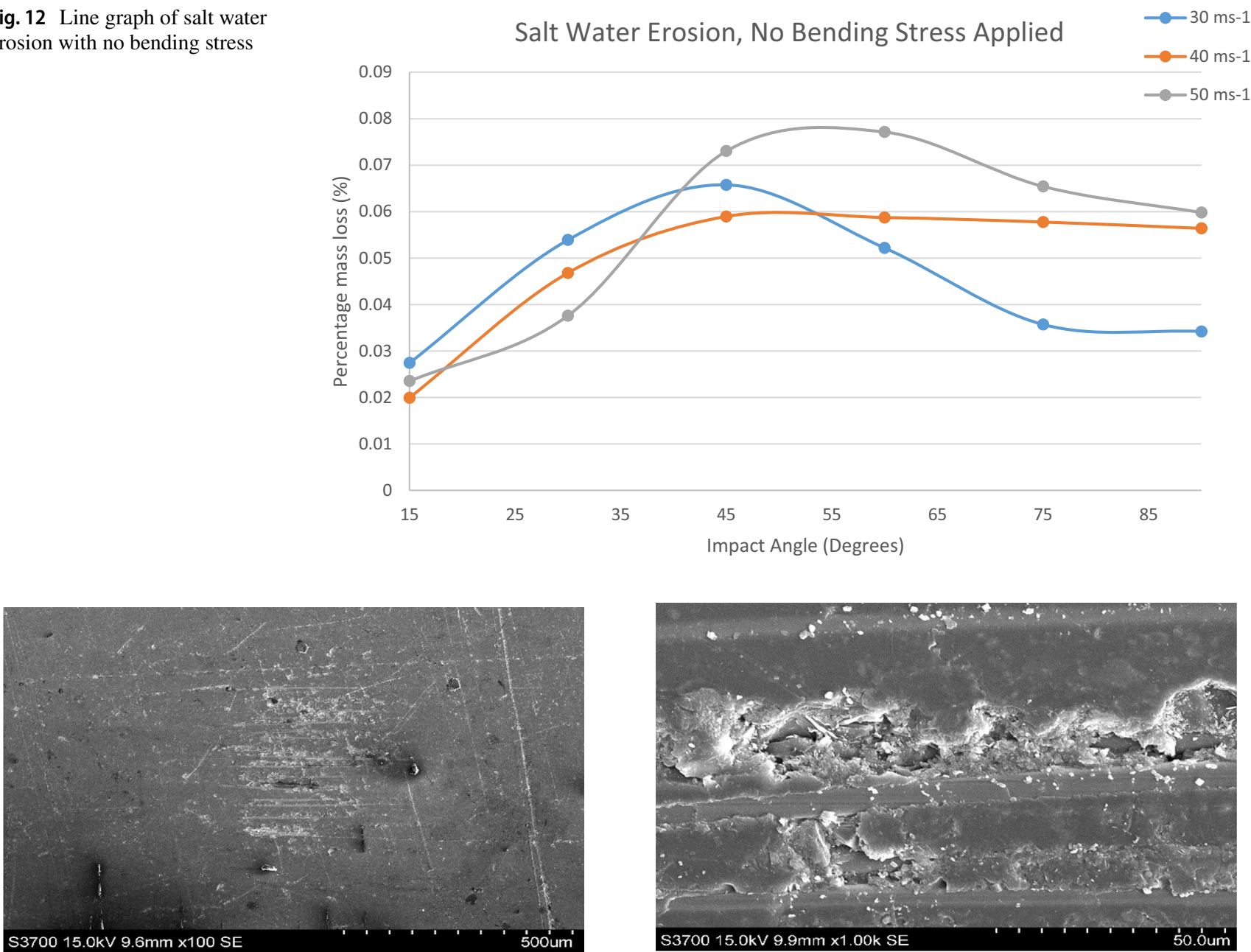

Fig. 13 SEM image of fresh water erosion on a stressed sample at $50 \mathrm{~ms}^{-1}, 30^{\circ}$

$V_{\mathrm{f}}=669.4 \mathrm{~g}=669.4 \mathrm{ml}$

$t=9629.4 \mathrm{~s}$

Rate $=\mathrm{W}_{\mathrm{i}}-$ W/t Rate $=0.377 \mathrm{ml} / \mathrm{s}$

In order to be able to bend the sample sufficiently a smaller size was chosen to obtain more deflection. This resulted in the unstressed sample size to be larger, this was compensated by reducing the mass loss by a correction factor according to the area in contact with the rain droplets.

\subsection{Limitations of the Rig}

The reason for the maximum running speed of $50 \mathrm{~ms}^{-1}$ and experimental distance of $108 \mathrm{~km}$ is due to the limitation of the experimental apparatus. The maximum running time of the rig is $36 \mathrm{~min}$ at the highest tangential velocity of $50 \mathrm{~ms}^{-1}$. This was also mentioned in a previous paper published using this same apparatus as the greater rotational speeds create an adverse amount of heat, which both change the operating
Fig. 14 SEM image of salt water erosion on a stressed sample at $50 \mathrm{~ms}^{-1}, 45^{\circ}$

conditions within the rig and also increase the chances of the output shaft of the motor to buckle and become potentially dangerous [14]. This limitation then determined the running time for all the experiments (Table 1).

$l=v \times t=60 \times(30 \times 60)=108,000 \mathrm{~m}$

\subsection{Material}

G10 epoxy glass laminate composite, manufactured by National Electrical Manufacture Association (NEMA) was used as a specimen. This is thermosetting industrial composite consists of continuous filament glass cloth as reinforcement and epoxy as the resin binder. Generally, G10 has high strength, low water absorption and good corrosion resistance in onshore and offshore environments. The mechanical and physical properties are as mentioned in Table 2 . 


\subsection{Post Processing}

After all the samples were weighed after testing, their changes in mass was formatted within excel and MATLAB to create a wear map and a contour map which both allow easy comparison of different conditions and the amount of erosion at each variable. To make this data from the experiments more relatable with the literature, the impact angle was changed from the angle of the sample holder which was used in the previous wear maps to the angle the droplet makes on the sample.

In comparison to the previous salt water experiments [13], during the removal of the samples from the rig, the samples were given a short wash in order to minimise the quantity of salt crystals on and within the sample.

For visual inspection an optical microscope was used to observe surface damage of the stressed samples, which proved to have some pros and cons against the SEM which was previously used. The optical microscope was much faster and easier to operate, and the sample was not required to have a gold coating. The absence of a gold coat was a great advantage as there are plans to retest the existing samples to investigate further erosion, this would not be possible if the sample was required to be gold plated. The SEM did however yield higher quality images and more detail was observed.

\section{Results}

\subsection{Wear Maps}

\subsubsection{Fresh Water Erosion with no Applied Bending Stress}

See Fig. 3.

\subsubsection{Fresh Water with Applied Bending Stress}

See Fig. 4.

\subsubsection{Salt Water with no Applied Bending Stress}

See Fig. 5.

\subsubsection{Salt Water Erosion with Applied Bending Stress}

See Fig. 6.

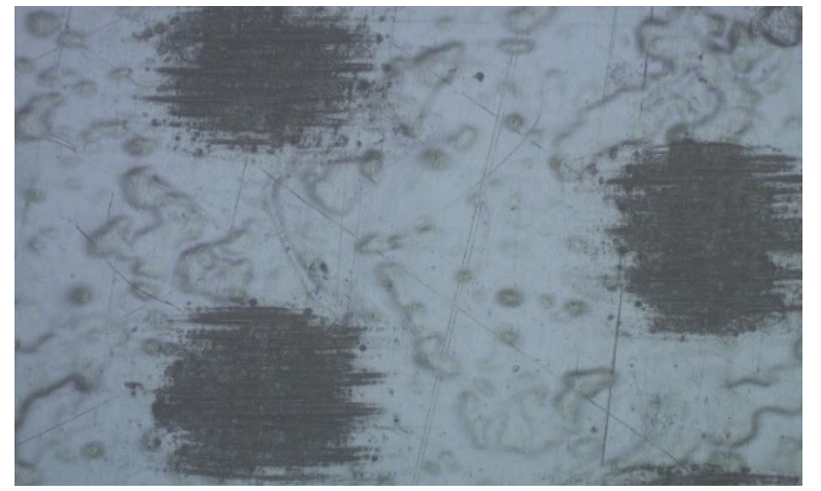

Fig. 15 Optical microscope image of unstressed sample at $50 \mathrm{~ms}^{-1}$, $30^{\circ}$

\section{Discussion}

\subsection{Unstressed Fresh Water Erosion}

The first experiment that was conducted was fresh water with no applied stress. From Fig. 3 the conditions that yielded the most erosion was at the maximum speed of $50 \mathrm{~ms}^{-1}$ and at droplet angle of $45^{\circ}$ with a mass loss of $0.11 \%$. From the wear map it is very distinguishable where the transition between negligible erosion to relatively high erosion occurs. This has been observed within the literature [15]. This is theorised to be the threshold number of droplets on the surface of the material sufficient to weaken it significantly to initiate erosion. This behaviour was observed with the majority of all the experiments tested. This was also observed in the SEM images of the samples as the erosion was concentrated in the areas where the fibres were closest to the surface and hence the top layer of epoxy was the thinnest (Figs. 7 and 8).

In the first experiment, i.e. fresh water with no applied stress, the most erosive impact angle range was approximately $45^{\circ}$ to $60^{\circ}$. This range of angles is interesting as classically ductile materials typically exhibit maximum erosion at relatively low angles of impact $\left(20-30^{\circ}\right)$ whereas with classically brittle materials the erosion increases continually until $90^{\circ}$ is reached [16]. This behaviour has been modelled using particle impacts; however the same behaviour has been observed using liquid impacts during these experiments.

The wear map from this experiment, Fig. 3, can also be visualised as a line graph shown in Fig. 9. From this graph it can be concluded that the material is neither experiencing fully ductile or fully brittle properties as the impact angle with the most erosion is $45^{\circ}$. This can be explained as G10 epoxy glass is a composite material involving a brittle glass fibre with a ductile matrix binding the fibres together. The surface features evident on the eroded samples are more in common with ductile gauging or ploughing type features rather than the fracturing or localised shattering that is 


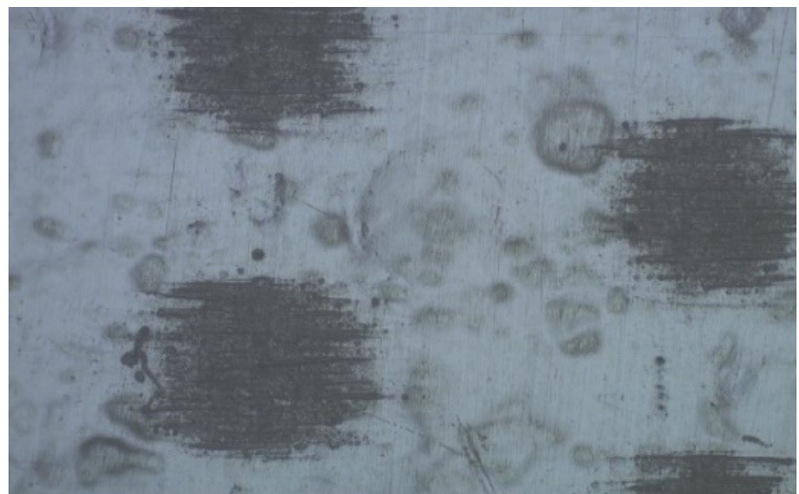

Fig. 16 Optical microscope image of unstressed Sample at $50 \mathrm{~ms}^{-1}$, $45^{\circ}$

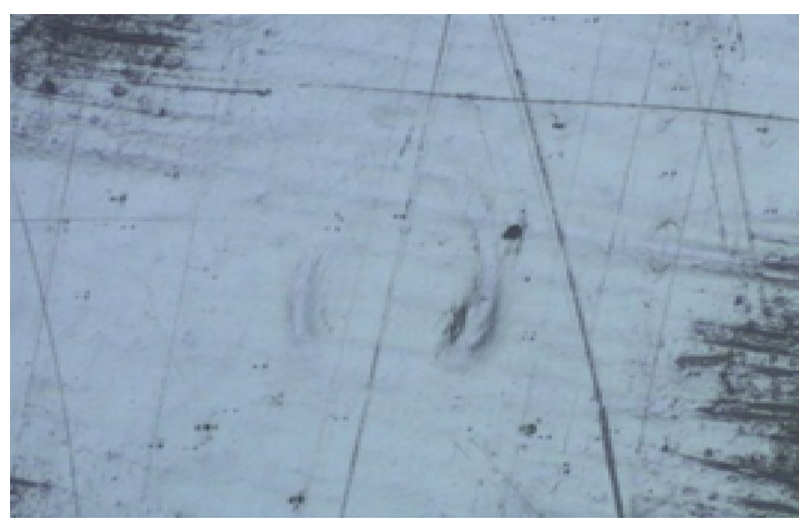

Fig. 17 Optical microscope image of stressed sample at $50 \mathrm{~ms}^{-1}$ at $60^{\circ}$

evident in brittle materials. [16] As a result, the behaviour could be classed as quasi-ductile. These results agree well with the work of Rafee Abdulmajeed Rafee Ahamed et al. [17] where tidal erosion was studied. It is interesting that although this work investigated erosion of G10 composite in slurries of sea water and sand ( $\mathrm{SiC}$ ), the impact angle exhibiting peak erosion is the same; thus, analogous behaviour was observed albeit in different conditions.

\subsection{Unstressed Salt Water Erosion}

The second experiment that was conducted was with 3.5\% salt water solution to simulate the effects of sea water on wind turbine blades under the same parameters to which the fresh water was tested. This was to determine if offshore wind turbines are eroding differently to those on shore. The Figs. 10 and 11 depict the erosion occurring to an extent where the glass fibres on the surface are exposed.

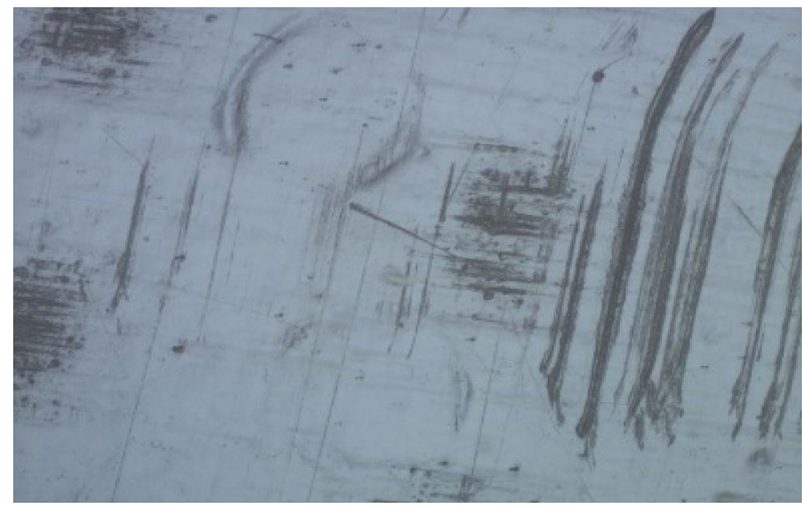

Fig. 18 Optical microscope image of stressed sample at $50 \mathrm{~ms}^{-1}, 45^{\circ}$

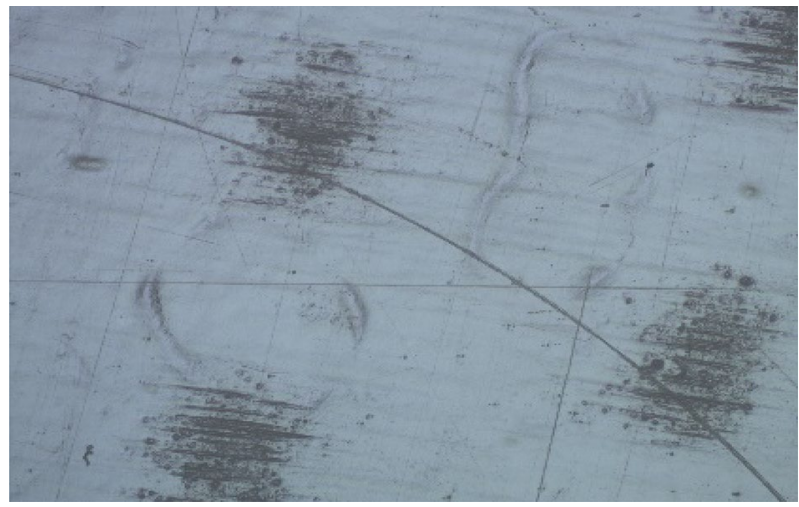

Fig. 19 Optical microscope image of stressed sample at $40 \mathrm{~ms}^{-1}, 45^{\circ}$

The maximum mass loss during this experiment was experienced at $50 \mathrm{~ms}^{-1}$ at $60^{\circ}$ with a total loss of $0.077 \%$; this can be seen in the wear map, Fig. 5. The difference in mass loss between the fresh water and salt water mass loss is not overly substantial as there will be some salt crystals remaining in the sample. From the wear map of the salt water testing (Fig. 5) a line graph can be constructed (Fig. 12). These results show neither fully "ductile" nor "brittle" erosion behaviour [16] as described in the literature in a similar fashion to the result exposed in fresh water.

\subsection{Stress Testing in Fresh Water and Salt Water Erosion}

The next tests that were conducted were a repeat of the fresh water and salt water experiments but under a static 3-point bend. This was to replicate the stresses which large wind turbine blades experience while in operation.

The maximum mass loss was at $50 \mathrm{~ms}^{-1}$ at 30 and $45^{\circ}$ with a $0.12 \%$ mass loss, this can be observed in the wear map in Fig. 5. This mass loss can be seen in the SEM images in Figs. 13 and 14 as the top layer of epoxy is eroded to the 

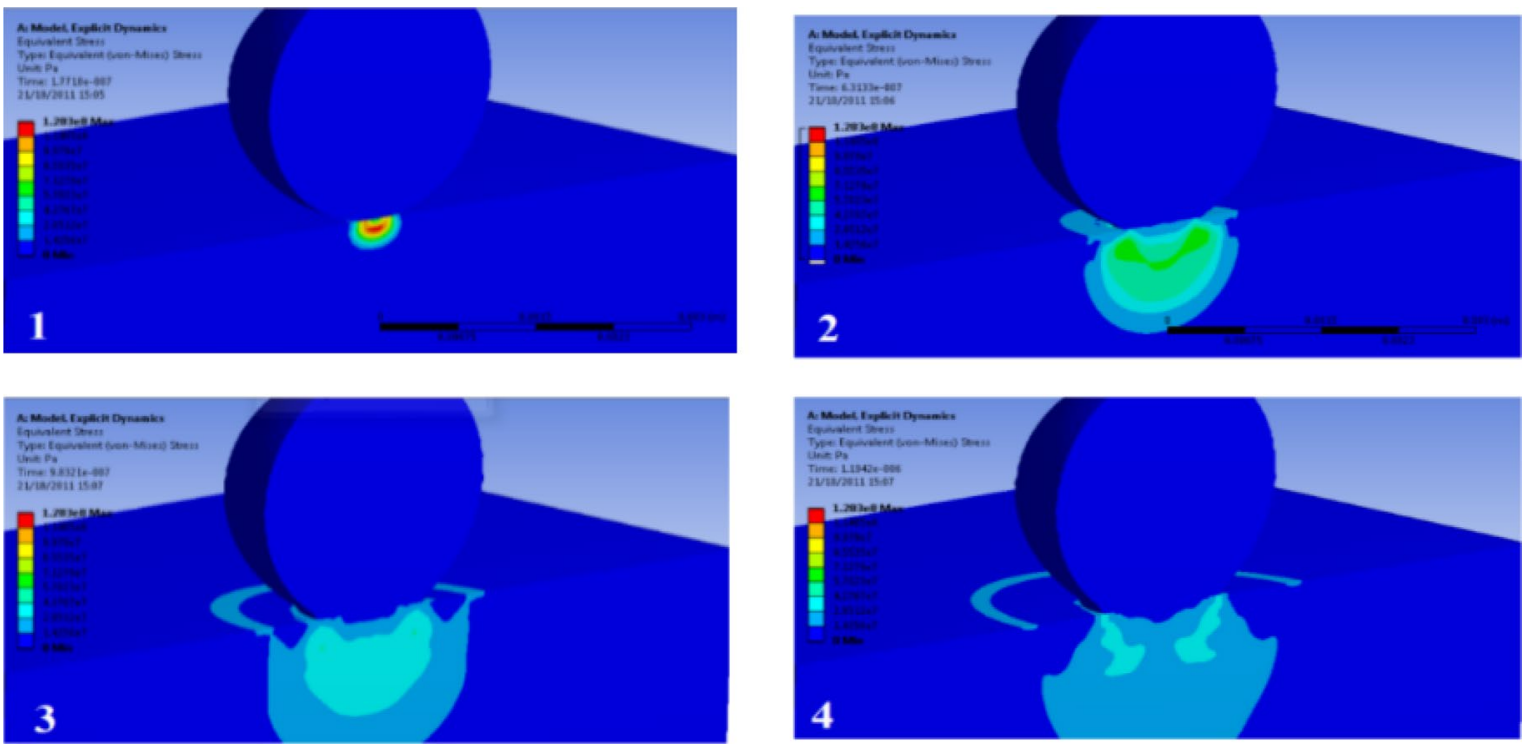

Fig. 20 Contour plot of sub surface forces [16]

Fig. 21 Comparison of erosion between the stressed and unstressed samples

\section{Comparison between stressed and un stressed samples}

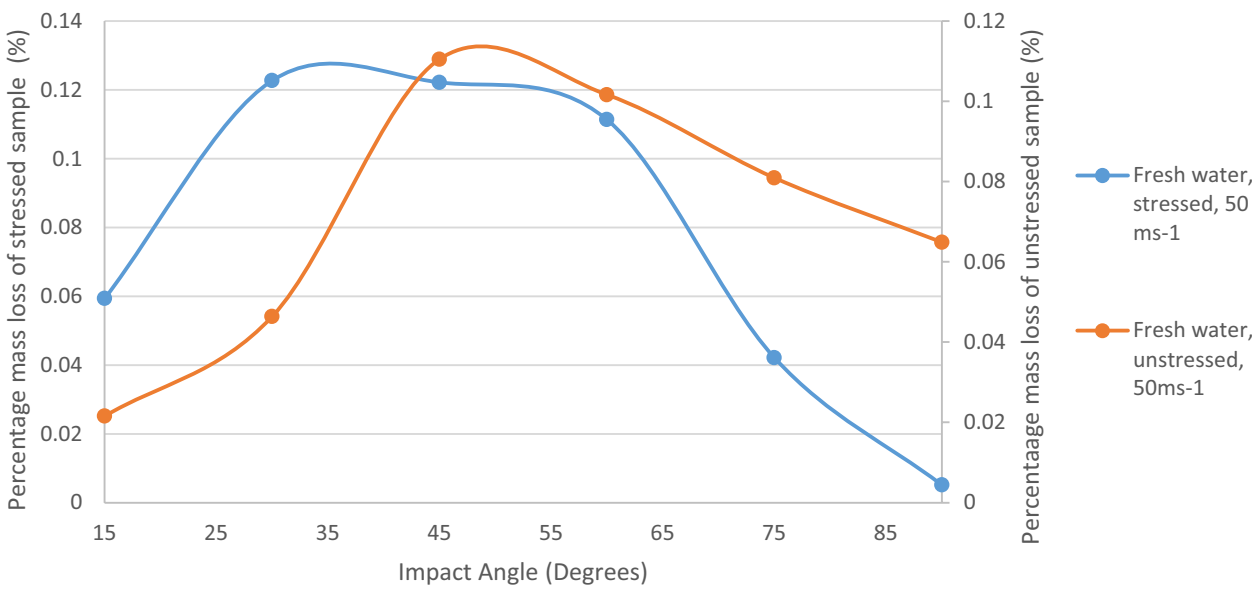

point where the fibres closest to the surface are exposed. The erosion is accelerated in this region due to the surface being slightly rougher at this point and the ploughing mechanism of the water droplet seems to have more effect on the surface by removing material. This is also observed in the optical microscope images of the unstressed sample (Figs. 15 and 16) as the point at which the fibres are closest to the surface show a greater dark area. This possibly implies that there is excessive pitting and erosion in the material.

From the wear maps in Figs. 4 and 6 a slightly unusual pattern occurred over the different impact angles. Instead of peaking at one impact angle this experiment showed two peaks which was not as expected.

The manner in which erosion occurs on the material from liquid impacts changes depending on the impact angle, in the same fashion as a particle impact. At lower impact angles the mechanism is more abrasion dominant resulting in shearing of the top surface; this can result in significant mass loss to the material, as once the top layer of the matrix is compromised, the fibres which are more brittle are exposed. This is seen in an SEM image Fig. 14. It is postulated that the high surface stresses influence the resultant damage caused by the liquid impact on the material. As a result the classical ductile to brittle crossover graph [16] for the stress test would be altered.

At higher impact angles, the water droplet induces a much larger force perpendicularly onto the sample resulting in sub surface cracks and brittle/fatigue type wear, potentially leading to delamination [18]. These two erosive mechanisms synergise to produce a distinctive pattern of erosion found 
in Figs. 3, 5, 9 and 12 which is consistent with the literature [16]. However when the sample is stressed it varies from this pattern.

The stressed samples showed different surface features in the optical microscope images as a circular ridge formation; this was not present in the optical microscope images of the unstressed samples (shown in Figs. 15 and 16). It is postulated that this is the result of the stresses on the material. The feature can be observed in the Figs. 17, 18 and 19.

It is assumed these features are associated with plastic deformations of the top surface due to the impact of a water droplets which can be termed SICD (Surface Impact Circular Deformation). In Fig. 17 there is a crack in the top surface to the right of the SICD; this was assumed to result from the stresses which would have occurred during the formation of the SICD. This crack could explain the slightly higher magnitude mass loss in the stress tests compared to the unstressed tests as this crack is liable to increase the erosion rate resulting in the glass fibres being at an increased risk of erosion which would lead to considerably more mass loss.

SICDs were only found on the stressed samples; this was possibly due to the sample being held under conditions closer to its yield stress and the effect of any additional stress on the sample by a liquid impact has more chance to cause plastic deformation of the top surface, resulting in a greater visible distribution to the surface. The shape of the SCIDs can be described from the shockwave that the droplet creates on the sample. This has been previously been investigated and modelled. Figure 20 shows a contour plot of von Mises Stress in the Epoxy plate during a $140 \mathrm{~ms}^{-1}$ impact, using a cross-sectional view to detail the stress dissipation beneath the surface of the plate [19].

From stages 3 and 4 of this model, a shockwave ring has formed around the droplet replicating the SICDs, therefor this could be the reasoning behind the formation.

A comparison between an unstressed and stressed sample can be shown in the line graph (Fig. 21), illustrating $50 \mathrm{~ms}^{-1}$ using fresh water as the erosive medium.

From this graph (Fig. 21), the stressed sample experiences high erosion over a larger range of impact angles and experiences erosion past threshold at lower angles of impact. Therefore, it can be deemed that the stressed sample is more susceptible to erosion than the unstressed sample. It is postulated that the stressed sample is experiencing a larger crossover between abrasion and brittle/fatigue erosion which allows the top surface of the material to be likely impeded and have fibre exposure. This behavioural change when stressed is theorised to be due to the additional stress from the droplet impact causing plastic deformation (as all stresses are additive). The graph suggests the sample is now behaving in a more "ductile" than "brittle" manner due to the slight shift to the left. This change could be due to the polymer (which is the more ductile portion of the material) governing the behaviour rather than the brittle glass fibres. Experimental evidence to support this would be the presence of SICDs on the surface, with these features indicative of ductile behaviour due to the applied stress.

During the salt water testing of the pre-stressed samples, the same pattern of a wider range of impact angles experiencing more considerable erosion was observed. This suggests similar mechanisms were involved for both exposure media in the above study.

\section{Conclusions}

i. Studies have indicated that there is a difference in material behaviour observed for rain erosion on a wind turbine blade when the material is unstressed compared to when the material has an applied load.

ii. The difference in behaviour is theorised to be due to the synergy between the initial stress plus the stress from the droplet impact, causing plastic deformation of the epoxy/polymer of the material when under stress.

iii. Changes in mechanical properties has had two effects on the material that were subject to testing. The first was higher erosion over a larger range of impact angles. This was due to a larger crossover between the abrasive mechanism and the brittle/fatigue mechanism by the droplet. The second was a different surface interference on the material due to the droplet impact which resulted in the creation of morphological feature on the top surface, Surface Impact Circular Deformation, (SICDs).

iv. It was concluded that for these studies, the erosive medium for liquid impact did not significantly affect the range of impact angles at which the highest erosion rates were observed.

Acknowledgements The authors would like to acknowledge the support of the Interreg (Northern Ireland-Ireland-Scotland) Special EU Programmes Grant No SPIRE2_INT - VA - 049 "'Storage Platform for the Integration of Renewable Energy (SPIRE 2)".

Open Access This article is distributed under the terms of the Creative Commons Attribution 4.0 International License (http://creativecommons.org/licenses/by/4.0/), which permits unrestricted use, distribution, and reproduction in any medium, provided you give appropriate credit to the original author(s) and the source, provide a link to the Creative Commons license, and indicate if changes were made. 


\section{References}

1. Igwemezie V, Mehmanparast A, Kolios A (2019) Current trend in offshore wind energy sector and material requirements for fatigue resistance improvement in large wind turbine support structuresa review. Renew Sustain Energy Rev 101:181-196

2. https://www.regjeringen.no/en/topics/energy/renewable-energy/ renewable-energy-production-in-norway/id2343462/. Accessed Oct 2018

3. Finjord F (2018) The impact of Norwegian-Swedish green certificate scheme on investment behaviour: a wind energy case study. Energy Policy 123:373-389

4. Renewable energy production in Norway (2016) https://www.dtu. dk/english/news/2018/03/dynamo-the-worlds-largest?id=00d42 9c3-eb5b-4e1a-8102-8016d0f15699. Accessed Sept 2018

5. Laursen J, Enevoldsen P, Hjort S (2007) 3D CFD quantification of the performance of a multi-megawatt wind turbine. J Phys 75:012007

6. Wang X, Wang L, Xia H (2015) Study on load and aerodynamic characteristics for rotating wind turbine rotor. In: 2015 IEEE International Conference on Mechatronics and Automation (ICMA), Beijing, pp. 1870-1874

7. World's longest wind turbine is put to the test (2018) https://www. lmwindpower.com/en/stories-and-press/stories/innovation/world s-longest-blade-is-put-to-the-test Accessed Nov 2018

8. UK climate (2018) https://www.metoffice.gov.uk/public/weather/ climate/Accessed Oct 2018

9. Macdonald H, Infield D, Nash DH, Stack MM (2016) Mapping hail meteorological observations for prediction of erosion in wind turbines. Wind Energy 19:777-784. https://doi.org/10.1002/ we. 1854

10. Whitelee Windfarm https://www.scottishpower.co.uk/whitelee/. Accessed Dec 2018
11. Keegan MH, Nash DH, Stack MM (2013) On erosion issues associated with the leading edge of wind turbine blades. J Phys D 46(38):383001

12. Sareen A, Sapre CA, Selig MS (2014) Effects of leading edge erosion on wind turbine blade performance. Wind Energy 17:1531-1542

13. Pugh K, Rasool G, Stack MM (2018) Some thoughts on mapping tribological issues of wind turbine blades due to effects of onshore and offshore raindrop erosion. J Bio Tribo Corros 4:50

14. Shokrieh MM et al (2004) Shear buckling of a composite drive shaft under torsion. Compos Struct 64(1):63-69

15. Amirzadeh B, Louhghalam A, Raessi M, Tootkaboni M (2017) A computational framework for the analysis of rain-induced erosion in wind turbine blades, part I: stochastic rain texture model and drop impact simulations. J Wind Eng Ind Aerodyn 163:33-43

16. Hutchings IM (1992) Tribology: friction and wear of engineering materials, vol 273. CRC Press, Boca Raton, pp 173-188

17. Ahamed RAR, Johnstone CM, Stack MM (2016) Impact angle effects on erosion maps of GFRP: applications to tidal turbines. J Bio- Tribo-Corros. 2(2): 14

18. Stachowiak GW, Batchelor AW (1993) Engineering tribology (tribology series) 24. Elsevier, Amsterdam, p 586

19. Keegan MH, Nash D, Stack M (2012) Modelling rain drop impact of offshore wind turbine blades. In: ASME Turbo Expo 2012, 2012-06-11-2012-06-15, Copenhagen

Publisher's Note Springer Nature remains neutral with regard to jurisdictional claims in published maps and institutional affiliations. 